\title{
IQGAP1, a negative regulator of cell-cell adhesion, is upregulated by gene amplification at 15q26 in gastric cancer cell lines HSC39 and 40A
}

Received: September 20, 2000 / Accepted: October 17, 2000

\begin{abstract}
Our previous comparative genomic hybridization (CGH) study revealed a novel amplified region at $15 \mathrm{q} 26$ in two cell lines established from diffuse types of gastric cancer (GC). In this amplified region, FES and IGFIR, known targets on $15 \mathrm{q} 26$, were located telomeric to the amplicon in the two cell lines, HSC39 and 40A, suggesting that another tumor-associated gene exists in this region. While screening expressed sequence tags (ESTs) for novel genes in this region, we identified the IQGAPI amplification. IQGAPI has been reported to encode a ras GAP-related protein, and its interaction with cadherin and/or $\beta$-catenin induces a dissociation of $\beta$-catenin from the cadherin-catenin complex, one of the mechanisms for cell-cell adhesion. Northern and Western blot analyses revealed that amplification of this gene was accompanied by corresponding increases in mRNA and protein expression. Moreover, immunocytochemical staining showed that overexpressed IQGAP1 accumulated at the membrane, suggesting its colocalization with $\beta$-catenin. Taken together, these findings suggest that IQGAPI may be one of the target genes in the $15 \mathrm{q} 26$ amplicon correlated with a malignant phenotype of gastric cancer cells, such as diffuse and invasive characteristics, through the disruption of E-cadherin-mediated cell-cell adhesion.
\end{abstract}

N. Sugimoto $\cdot$ I. Imoto $\cdot$ Y. Fukuda $\cdot$ N. Kurihara $\cdot$ J. Inazawa $(\square)$ Department of Molecular Cytogenetics, Division of Genetics,

Medical Research Institute, Tokyo Medical and Dental University,

1-5-45 Yushima, Bunkyo-ku, Tokyo 113-8519, Japan

Tel. +81-3-5803-5820; Fax +81-3-5803-5820

e-mail: johinaz.cgen@mri.tmd.ac.jp

N. Sugimoto $\cdot$ R. Kamiyama

School of Allied Health Science, Faculty of Medicine, Tokyo

Medical and Dental University, Tokyo, Japan

S. Kuroda $\cdot$ K. Kaibuchi

Division of Signal Transduction, Nara Institute of Science and

Technology, Ikoma, Japan

A. Tanigami

Otsuka GEN Research Institute, Otsuka Pharmaceutical Co., Ltd,

Tokushima, Japan
Key words Gastric cancer · $15 \mathrm{q} 26$ - Gene amplification · $I Q G A P 1 \cdot \beta$-Catenin

\section{Introduction}

Evidence indicates that the development and progression of gastric cancer (GC) is caused by multistep genetic changes, such as gene amplification (Tahara 1995). Recently, we identified novel amplifications in different chromosomes by comparative genomic hybridization $(\mathrm{CGH})$ in $25 \mathrm{GC}$ cell lines (Fukuda et al. 2000). Among these, a novel amplicon at 15 q26 was detected in two cell lines, HSC39 and 40A, established from a patient with signet-ring cell carcinoma, using different methods (Yanagihara et al. 1991). Amplifications at $15 \mathrm{q} 26$ have been found in various malignancies (Knuutila et al. 1999), including breast cancers (Muleris et al. 1994) and other types of tumors (Zhang et al. 1993; Armengol et al. 2000). In this region, FES and/or IGFIR have been identified as targets for gene amplifications in breast cancer (Berns et al. 1992; Almeida et al. 1994), melanoma (Zhang et al. 1993), and pancreatic adenocarcinoma (Armengol et al. 2000). In contrast, we found that both genes were located telomeric to the amplicon at $15 \mathrm{q} 26$ in HSC39 and 40A, suggesting that another tumor-associated gene is likely to exist in this region. In order to identify novel tumor-associated gene(s) from this telomeric amplicon, we screened expressed sequence tags (ESTs) mapped on $15 \mathrm{q} 26$, and found that IQGAPI may be one of the target genes for the 15q26-amplicon in these two cell lines.

\section{Materials and methods}

Tumor cell lines

All of the $25 \mathrm{GC}$ cell lines previously analyzed by CGH (Fukuda et al. 2000) were maintained in RPMI 1640 
medium supplemented with $10 \%$ fetal calf serum (FCS) and penicillin-streptomycin. Of the cell lines, HSC39 and 40A were a gift from Dr. K. Yanagihara.

\section{Fluorescence in situ hybridization (FISH)}

To reduce the size of the amplicon at $15 \mathrm{q} 26$ in HSC39, we performed FISH, using yeast artificial chromosomes (YACs) and P1-derived artificial chromosome (PAC) clones, as previously described (Inazawa et al. 1993). Twenty-nine YACs (797-E-2, 893-A-2, 929-C-7, 826-A-8, 802-B-4, 781-G-3, 851-E-2, 964-F-8, 881-C-4, 765-H-1, 966A-2, 747-A-12, 759-H-8, 739-D-8, 883-C-4, 876-A-1, 787-E2, 932-G-12, 911-B-8, 748-H-5, 744-B-7, 803-H-4, 838-H-8, 943-B-4, 859-A-12, 933-B-5, 757-H-6, 771-E-6, 914-F-1), archived by the Whitehead Institute Genome Center (http://www-genome.wi.mit.edu/) and PACs (17n9 containing the FES gene, 443n8 containing the IQGAP1 gene) were chosen as probes. We examined the number of FISH signals specific to each probe in 10 to 15 metaphase chromosomes of each cell line. Hybridization to normal lymphocyte nuclei was performed as a control to ascertain that the probes recognized a single-copy target.

\section{Southern and Northern blot hybridization}

To identify EST clones within the 15q26 amplicon, we carried out Southern blot analysis, as described elsewhere (Sakabe et al. 1999). The following EST clones mapped around this amplicon were used as probes in this experiment: GEN-012E10, GEN-326C09, GEN-178E08, GEN024G06, GEN-134B06, GEN-093E03, and GEN-154A06, based upon the GENOTK human cDNA database (http://genotk.genome.ad.jp). Ten micrograms of DNA from each cell line or normal peripheral leukocytes (control) was separated with $0.8 \%$ agarose gel and transferred to a nylon membrane. Each probe was labeled with $\left[\alpha-{ }^{32} \mathrm{P}\right]$ $\mathrm{dCTP}$, using a random priming method, and hybridized to the prehybridized membranes. Because a distinct amplification was detected with two cDNAs of GEN-326C09 and 154A06, we then performed Northern blot analysis to investigate the expression status of these genes. Ten micrograms of total RNA from each cell line was electrophoresed in $1 \%$ agarose $/ 0.67 \mathrm{M}$ formaldehyde gel, and then transferred to a nylon membrane. Membranes were hybridized with each $\left[\alpha-{ }^{32} \mathrm{P}\right] \mathrm{dCTP}$-labeled probe.

\section{DNA sequencing}

cDNAs were sequenced using a $377 \mathrm{ABI}$ autosequencer (Applied Biosystems, Tokyo, Japan).

\section{Western blot analysis}

Cultured cells were lysed with RIPA buffer $(10 \mathrm{mM}$ Tris$\mathrm{HCl}$ [pH7.4], $150 \mathrm{mM} \mathrm{NaCl}, 1 \%$ Triton $\mathrm{X}-100,0.1 \%$ sodium dodecylsulfate [SDS], $1 \%$ sodium deoxycholate, and $1 \mathrm{mM}$ phenylmethanesulfonyl fluoride [PMSF]) for Western blot analysis. Cell lysates were separated by $8 \%$ SDSpolyacrylamide gel electrophoresis (PAGE) (Laemmli 1970) and electrotransfered to polyvinylidene difluoride (PVDF) membranes (Fukata et al. 1997). After transfer, membranes were washed and incubated with blocking buffer $(5 \%$ non-fat dry milk, Tris-buffered saline [TBS] [pH7.6], 0.1\% Tween 20 overnight at $4^{\circ} \mathrm{C}$. Anti-human IQGAP1 polyclonal antibody was used as the primary antibody (Kuroda et al. 1996). Subsequently, horseradish peroxidase-conjugated sheep anti-rabbit $\mathrm{Ig}$ served as the secondary antibody for the enhanced chemiluminescence (ECL) detection system (Amersham Pharmacia Biotech, Tokyo, Japan).

\section{Immunofluorescence cytochemistry}

Cultured cells were fixed for immunostaining in phosphatebuffered saline (PBS), containing $4 \%$ paraformaldehyde and blocked with antibody dilution buffer $(1 \%$ bovine serum albumin [BSA] in PBS) for $30 \mathrm{~min}$ at room temperature, and then incubated for $60 \mathrm{~min}$ at room temperature with anti-human IQGAP1 polyclonal antibody (Kuroda et al. 1996) or anti- $\beta$-catenin monoclonal antibody (Transduction Laboratories, Lexington, KY, USA) as the first antibody at $10 \mu \mathrm{g} / \mathrm{ml}$. Binding was detected by fluorescein isothiocyanate (FITC)-conjugated anti-rabbit or mouse IgG (MBL, Nagoya, Japan) diluted 1:100 with antibody dilution buffer, and incubated for $45 \mathrm{~min}$ at room temperature. The cells were observed under a fluorescence microscope (Olympus, Tokyo, Japan) equipped with a cooled-charged devised camera.

\section{In-vitro binding assay}

The interactions of wild type- or mutant type-MBP- $\beta$ catenin with glutathione-s-transterase (GST)-IQGAP1 or GST- $\alpha$-catenin were examined as described (Fukata et al. 1997). Briefly, to obtain GST fusion protein, cDNA fragments of IQGAP1 or $\alpha$-catenin were subcloned into pGEX2T or pGEX4T-1, respectively, whereas, to obtain MBP- $\beta$-catenin, the wild type or mutant form (deletion of 28 to 134 amino acids) of $\beta$-catenin was subcloned into $\mathrm{pMal}$ $\mathrm{C}-2$. The expression and purification of various GST and maltose binding protein (MBP) fusion proteins were done as described elsewhere (Fukata et al. 1997; Kuroda et al. 1998). MBP- $\beta$-catenin constructs $(50 \mathrm{nM}$ each) were mixed with affinity beads coated with GST, GST-IQGAP1, or GST- $\alpha$ catenin ( $40 \mathrm{pmol}$ each) in buffer $\mathrm{A}(20 \mathrm{mM}$ Tris- $\mathrm{HCl}$ [pH7.4], $1 \mathrm{mM}$ ethylene diamine tetraacetic acid [EDTA], $1 \mathrm{mM}$ dithiothreitol, $500 \mathrm{mM} \mathrm{NaCl}, 0.1 \%$ Triton X-100, $0.1 \%$ 3-[(3-cholamidopropyl)dimethylammonio]-1-propanesulfonate [CHAPS], $10 \mu \mathrm{M}$ phenylmethylsulfonyl fluoride [PMSF], $10 \mu \mathrm{g} / \mathrm{ml}$ leupeptin). The beads were then washed with buffer A, and the bound proteins were coeluted with GST fusion proteins by the addition of buffer A containing $10 \mathrm{mM}$ reduced glutathione. The eluates were subjected to 
Fig. 1. Amplicon map of the $15 \mathrm{q} 26$ region in the gastric cancer (GC) cell line HSC39. The upper scale shows the ordering of sequence-tagged site (STS) markers mapped on this region (http://www.ncbi.nlm.nih gov/genemap99/ and http://wwwgenome.wi.mit.edu/). Yeast artificial chromosomes (YACs) and P1-derived artificial chromosome $(P A C)(17 \mathrm{n} 9$ containing the FES gene) anchored to the markers are represented as bars. Based on fluorescence in situ hybridization (FISH) analyses, using YACs and the PAC clone as probes, the homogeneous staining region (HSR) is indicated in relation to genetic markers between GATA88C02 and D15S946, and open bars represent YAC clones that showed amplification patterns in FISH analyses. Below, typical FISH images show HSR with 759-H-8 YAC, while PAC pDJ17n9, which contains the FES gene localized outside the amplicon, did not show any amplification
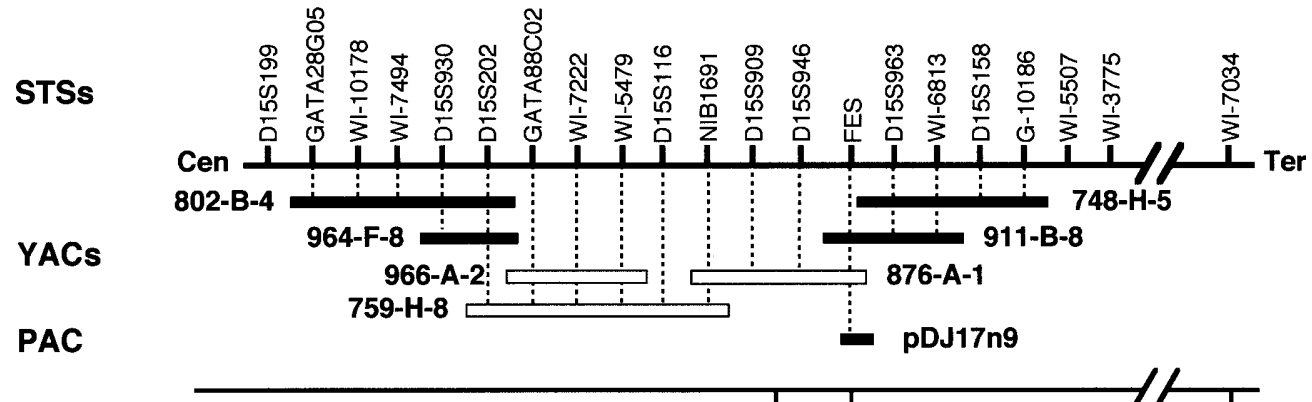

Genes

FISH images

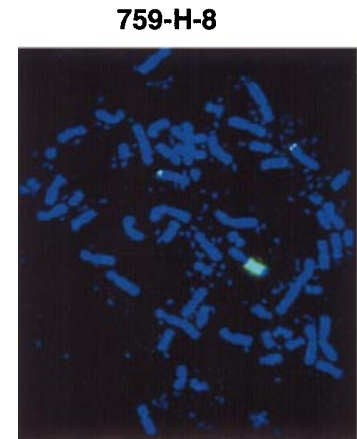

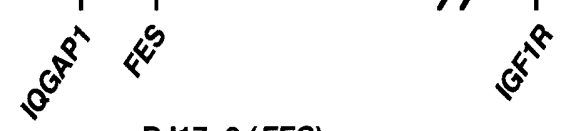

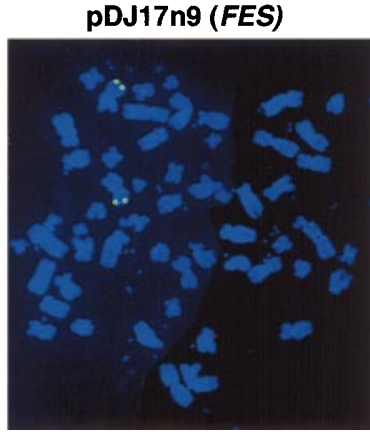

Fig. 2. Representative results of Southern- (A), Northern- (B), and Western-blot analyses (C) as well as immunofluorescence cytochemistry (D) of IQGAP1 in GC cell lines. Two cell lines (HSC39 and HSC40A) had demonstrated amplification patterns in our comparative genomic hybridization $(\mathrm{CGH})$ analysis reported elsewhere (Fukuda et al. 2000). Note that amplification and overexpression of $I Q G A P 1$ were observed in these two cell lines $(\mathbf{A}, \mathbf{B}, \mathbf{C})$. Immunofluorescence cytochemical analysis in the HSC39 cell line suggested that overexpressed IQGAP1 and $\beta$-catenin might be colocalized along the cell membrane $(\mathbf{D})$

A. Southern blot

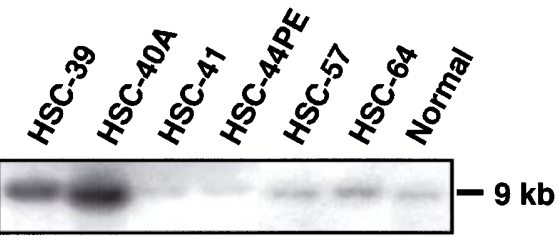

B. Northern blot

IQGAP1

GAPDH

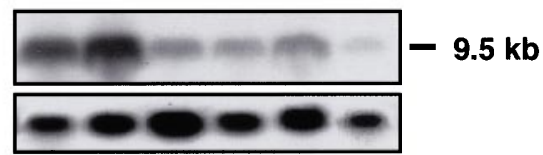

C. Western blot

IQGAP1

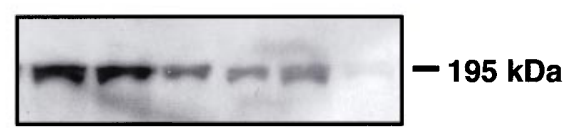

D. Immunocytochemistry

IQGAP1

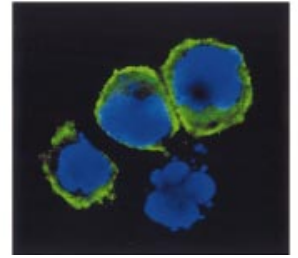

$\beta$-catenin

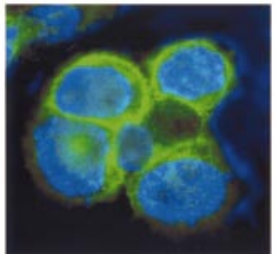


SDS-PAGE, followed by immunoblotting with anti-MBP antibody (Fukata et al. 1996).

\section{Results and discussion}

Amplification of $15 \mathrm{q} 26$ has been reported in various types of malignancy, such as lymphomas, non-small-cell and small-cell lung cancers, head and neck squamous cell carcinomas, and breast cancer (Knuutila et al. 1999), suggesting that the 15q26-amplicon harbors the gene(s) responsible for tumorigenesis in a broad spectrum of tumors. Therefore, characterization of the 15q26-amplicon could help us to identify candidate(s) for target genes from this region. In order to determine the smallest amplicon in HSC39 exhibiting gains in CGH analysis, we performed FISH with 29 YACs as probes mapped within 15q25-26. As shown in Fig. 1 , one marker chromosome exhibited a homogeneous staining region (HSR) signal for YACs between GATA88C02 and D15S946 at $15 \mathrm{q} 26$ in $\mathrm{HSC} 39$, and the region was narrowed down to about $2 \mathrm{Mb}$, based on sequence-tagged sites (STSs) and the sizes of YACs. FISH analyses using PACs containing FES mapped at $15 \mathrm{q} 26$ revealed no increase in copy number. Because IGF1R has been mapped to a site telomeric to FES (GeneMap99; http://www.ncbi. nlm.nih.gov/genemap99/), both genes were located telomeric to the 15q26-amplicon in HSC39 (Fig. 1), suggesting that another tumor-associated gene exists in this amplified region of GC cell lines.

To identify target genes in this amplicon, we first screened ESTs localized to 15q25-26, using Southern blot analysis, in 25 GC cell lines (Fukuda et al. 2000). Among them, GEN-326C09 and GEN-154A06 showed amplifications in two cell lines, HSC39 and HSC40A (Fig. 2). By contrast, probes for other genes outside this amplicon, such as IGF1R and FES, revealed no amplifications (data not shown). Northern blot analysis in these two EST clones clearly demonstrated that both transcripts were the same size and were upregulated by a mechanism of gene amplification in HSC39 and HSC40A, which exhibited distinct amplifications of these two ESTs on Southern blot (Fig. 2). A homology search, using the FASTA program (Pearson and Lipman 1988), showed that the nucleotide sequences of these two cDNAs were identical to parts of a known gene, IQGAP1 (gdb: NM_003870). Moreover, overexpression of $I Q G A P 1$ in cell lines was confirmed at the protein level by Western blot analysis (Fig. 2), suggesting that IQGAPI is one of target genes for the 15q26-amplicon in HSC39 and $40 \mathrm{~A}$.

IQGAPI encodes a ras GAP-related protein containing the IQ motif (Weissbach et al. 1994; Kuroda et al. 1996). IQGAP1 protein has been reported to accumulate at cell-cell contact sites in an E-cadherin and/or $\beta$-catenin dependent manner. This molecule directly interacts with $\beta$-catenin and the cytoplasmic domain of E-cadherin both in vitro and in vivo (Fukata et al. 1999). These findings are in keeping with our results of immunofluorescence cytochemical analysis using IQGAP1 and $\beta$-catenin antibodies, which

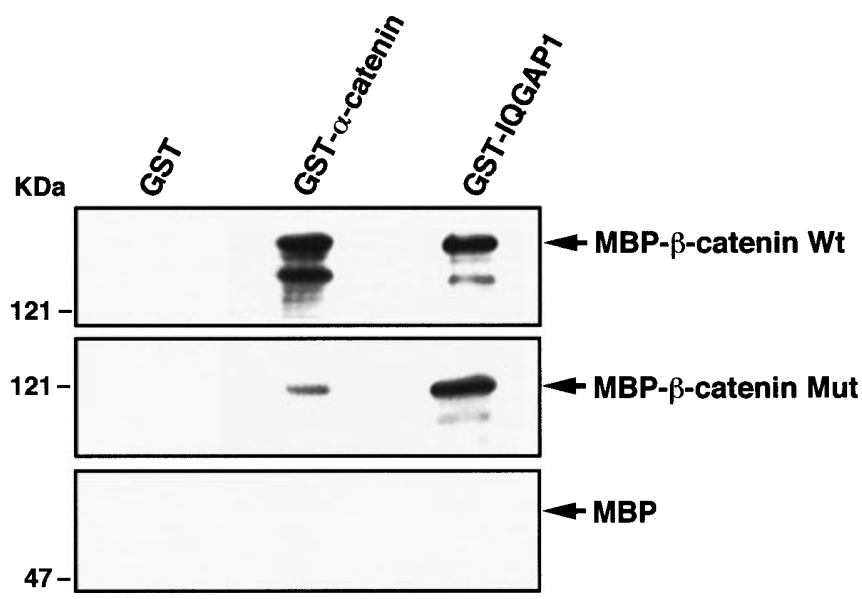

Fig. 3. Representative results of in-vitro binding assay between $\beta$ catenin and IQGAP1 or $\alpha$-catenin. The arrows indicate wild-type (Wt) of MBP- $\beta$-catenin, mutant type (Mut) of MBP- $\beta$-catenin (deletion of 28 to 134 amino acids) or MBP alone (negative control). Both GSTIQGAP1 and GST- $\alpha$-catenin were capable of binding to the wild type of MBP- $\beta$-catenin. Note that GST-IQGAP1 can interact with mutant type of MBP- $\beta$-catenin to the same extent as wild type, whereas GST$\alpha$-catenin cannot

suggested the colocalization of both proteins along the cell membrane in HSC39 (Fig. 2). IQGAP1, which contains the $\alpha$-catenin-binding domain (Aberle et al. 1994), interacts with the amino acid-terminus of $\beta$-catenin, (Fukata et al. $1999)$ dissociating $\alpha$-catenin from the E-cadherin $/ \beta$-catenin complex in vitro (Fukata et al. 1999). Because the association of $\alpha$-catenin with the E-cadherin $/ \beta$-catenin complex, through interaction with $\beta$-catenin, is essential for $\mathrm{E}$ cadherin activity, including cell-cell adhesion, IQGAP1 is thought to act as a negative regulator of cadherin function.

Alteration of the E-cadherin-mediated cell adhesion system is an important contributory mechanism in tumor pathogenesis (Hirohashi 1998). Dissociation of $\alpha$-catenin from the E-cadherin/ $\beta$-catenin complex may confer invasiveness and metastatic potential to tumor cells, due to disruption of the E-cadherin-mediated cell adhesion system. Indeed, abnormal E-cadherin expression (which may induce disruption of its normal function) caused by mutations or promoter hypermethylations has been reported to be responsible for the scattered phenotype of diffuse type of gastric cancer (Becker et al. 1994; Tamura et al. 2000). Accordingly, overexpression of IQGAPI may be an another mechanism for the dissociation of $\alpha$-catenin from the E-cadherin/ $\beta$-catenin complex, resulting in disruption of the E-cadherin-mediated cell adhesion system.

Interestingly, in HSC39 cells - one of the cell lines that overexpressed IQGAP1 - impaired cell-cell adhesion and the complete abolition of E-cadherin function have been reported (Oyama et al. 1994; Kawanishi et al. 1995; Jawhari et al. 1999). One of the mechanisms of this disruption of $E$ cadherin function is due to $\beta$-catenin mutation, resulting in protein truncation with loss of the $\alpha$-catenin-binding site (Kawanishi et al. 1995). However, the deleted region of $\beta$ catenin in HSC39 is from amino acids 28 to 134, which 
overlaps with neither the IQGAP1-binding site (1-183) nor the $\alpha$-catenin-binding site (120-151) (Aberle et al. 1994; Fukata et al. 1999). Our preliminary study of the binding of $\beta$-catenin to $\alpha$-catenin indicated that truncated type $\beta$ catenin had weak binding activity for $\alpha$-catenin, although the binding was not completely disrupted (Fig. 3). In addition, there was no difference in the binding of $\beta$-catenin to IQGAP1 between truncated type $\beta$-catenin and wild type $\beta$ catenin (Fig. 3). Therefore, overexpressed IQGAP1 due to gene amplification and truncated $\beta$-catenin may synergistically affect the activity of $\beta$-catenin to bind $\alpha$-catenin, resulting in disruption of the E-cadherin-mediated cell adhesion system in these cell lines. The pathological roles of IQGAP1 in gastric carcinogenesis in vivo, particularly the diffuse type, should be clarified in future studies.

Acknowledgments We thank Dr Kazuyoshi Yanagihara for the kind gift of HSC39 and 40A, and Professor Yusuke Nakamura for his continuous encouragement. This work was supported by Grants-in-Aid from the Ministry of Health and Welfare of Japan, the Ministry of Education, Science, Sports, and Culture of Japan, and the Organization for Pharmaceutical Safety and Research (OPSR); and in part by a grant from the Novartis Foundation (Japan) for the Promotion of Science.

\section{References}

Aberle H, Butz S, Stappert J, Weissig H, Kemler R, Hoschuetzky H (1994) Assembly of the cadherin-catenin complex in vitro with recombinant proteins. J Cell Sci 107:3655-3663

Almeida A, Muleris M, Dutrillaux B, Malfoy B (1994) The insulin-like growth factor I receptor gene is the target for the $15 \mathrm{q} 26$ amplicon in breast cancer. Genes Chromosomes Cancer 11:63-65

Armengol G, Knuutila S, Lluis F, Capella G, Miro R, Caballin MR (2000) DNA copy number changes and evaluation of MYC, IGF1R, and FES amplification in xenografts of pancreatic adenocarcinoma. Cancer Genet Cytogenet 116:133-141

Becker KF, Atkinson MJ, Reich U, Becker I, Nekarda H, Siewert JR, Hofler H (1994) E-cadherin gene mutations provide clues to diffuse type gastric carcinomas. Cancer Res 54:3845-3852

Berns EM, Klijn JG, van Staveren IL, Portengen H, Foekens JA (1992) Sporadic amplification of the insulin-like growth factor 1 receptor gene in human breast tumors. Cancer Res 52:1036-1039

Fukata M, Kuroda S, Fujii K, Nakamura T, Shoji I, Matsuura Y, Okawa K, Iwamatsu A, Kikuchi A, Kaibuchi K (1997) Regulation of cross-linking of actin filament by IQGAP1, a target for cdc42. J Biol Chem 272:29579-29583

Fukata M, Kuroda S, Nakagawa M, Kawajiri A, Itoh N, Shoji I, Matsuura Y, Yonehara S, Fujisawa H, Kikuchi A, Kaibuchi K (1999) Cdc42 and Rac1 regulate the interaction of IQGAP1 with betacatenin. J Biol Chem 274:26044-26050

Fukuda Y, Kurihara N, Imoto I, Yasui K, Yoshida M, Yanagihara K, Park J-G, Nakamura Y, Inazawa J (2000) CD44 is a potential target of amplification within the $11 \mathrm{p} 13$ amplicon detected in four of 25 gastric-cancer cell lines. Genes Chromosomes Cancer 29:315-324

Hirohashi S (1998) Inactivation of the E-cadherin-mediated cell adhesion system in human cancers. Am J Pathol 153:333-339
Inazawa J, Saito H, Ariyama T, Abe T, Nakamura Y (1993) High resolution cytogenetic mapping of 342 markers including 43 RFLP markers on human chromosome 17 by fluorescence in situ hybridization. Genomics 17:153-162

Jawhari AU, Noda M, Farthing MJ, Pignatelli M (1999) Abnormal expression and function of the E-cadherin-catenin complex in gastric carcinoma cell lines. Br J Cancer 80:322-330

Kawanishi J, Kato J, Sasaki K, Fujii S, Watanabe N, Niitsu Y (1995) Loss of E-cadherin-dependent cell-cell adhesion due to mutation of the $\beta$-catenin gene in a human cancer cell line, HSC-39. Mol Cell Biol 15:1175-1181

Knuutila S, Aalto Y, Autio K, Bjorkqvist AM, El-Rifai W, Hemmer S, Huhta T, Kettunen E, Kiuru-Kuhlefelt S, Larramendy ML, Lushnikova T, Monni O, Pere H, Tapper J, Tarkkanen M, Varis A, Wasenius VM, Wolf M, Zhu Y (1999) DNA copy number losses in human neoplasms. Am J Pathol 155:683-694

Kuroda S, Fukata M, Kobayashi K, Nakafuku M, Nomura N, Iwamatsu A, Kaibuchi K (1996) Identification of IQGAP as a putative target for the small GTPases, Cdc42 and Rac1. J Biol Chem 271:2336323367

Kuroda S, Fukata M, Nakagawa M, Fujii K, Nakamura T, Ookubo T, Izawa I, Nagase T, Nomura N, Tani H, Shoji I, Matsuura Y, Yonehara S, Kaibuchi K (1998) Role of IQGAP1, a target of the small GTPases Cdc42 and Rac1, in regulation of E-cadherinmediated cell-cell adhesion. Science 281:832-835

Laemmli UK (1970) Cleavage of structural proteins during the assembly of the head of bacteriophage T4. Nature 227:680-685

Muleris M, Almeida A, Gerbault-Seureau M, Malfoy B, Dutrillaux B (1994) Detection of DNA amplification in 17 primary breast carcinomas with homogeneously staining regions by a modified comparative genomic hybridization technique. Genes Chromosomes Cancer 10:160-170

Oyama T, Kanai Y, Ochiai A, Akimoto S, Oda T, Yanagihara K, Nagafuchi A, Tsukita S, Shibamoto S, Ito F, Takeichi M, Matsuda H, Hirohashi S (1994) A truncated beta-catenin disrupts the interaction between E-cadherin and alpha-catenin: a cause of loss of intercellular adhesiveness in human cancer cell lines. Cancer Res 54:62826287

Pearson WR, Lipman W (1988) Improved tools for biological sequence comparison. Proc Natl Acad Sci USA 85:2444-2448

Sakabe T, Shinomiya T, Mori T, Ariyama Y, Fukuda Y, Fujiwara T, Nakamura Y, Inazawa J (1999) Identification of a novel gene, MASL1, within an amplicon at 8p23.1 detected in malignant fibrous histiocytomas by comparative genomic hybridization. Cancer Res 59:511-515

Tahara E (1995) Molecular biology of gastric cancer. World J Surg 19:484-490

Tamura G, Yin J, Wang S, Fleisher AS, Zou T, Abraham JM, Kong D, Smolinski KN, Wilson KT, James SP, Silverberg SG, Nishizuka S, Terashima M, Motoyama T, Meltzer SJ (2000) E-Cadherin gene promoter hypermethylation in primary human gastric carcinomas. J Natl Cancer Inst 92:569-573

Weissbach L, Settleman J, Kalady MF, Snijders AJ, Murthy AE, Yan YX, Bernards A (1994) Identification of a human rasGAP-related protein containing calmodulin-binding motifs. J Biol Chem 269:20517-20521

Yanagihara K, Seyama T, Tsumuraya M, Kamada N, Yokoro K (1991) Establishment and characterization of human signet ring cell gastric carcinoma cell lines with amplification of the c-myc oncogene. Cancer Res 51:381-386

Zhang J, Trent JM, Meltzer PS (1993) Rapid isolation and characterization of amplified DNA by chromosome microdissection: identification of IGF1R amplification in malignant melanoma. Oncogene $8: 2827-2831$ 